\title{
Perforación espontánea de colédoco en un paciente pediátrico. Presentación de caso y revisión breve de la literatura
}

\author{
Spontaneous perforation of the common bile duct in a pediatric patient. Case report and \\ short review of the literature
}

Cristhian G. Godínez-Borrego*, Sergio Velasco-Villanueva y Jorge A. Mújica-Guevara

Servicio de Cirugía Pediátrica, Hospital General Regional No. 1, Instituto Mexicano del Seguro Social, Santiago de Querétaro, Querétaro, México

\begin{abstract}
Resumen
Introducción: La perforación espontánea del colédoco es rara. La causa generalmente se desconoce, aunque en ocasiones guarda relación con una malformación de la vía biliar. Caso clínico: Niña de 1 año, con distensión abdominal, hiporexia y fiebre, tomografía con ascitis, se interviene y se encuentra bilioma retroperitoneal, peritonitis y perforación de colédoco. Se realiza resección en bloque y anastomosis hepatoduodenal primaria. Discusión: La perforación espontánea del colédoco tiende a evolucionar insidiosamente y el retraso en el diagnóstico es frecuente. El manejo quirúrgico es resolutivo; existen distintas técnicas descritas. Conclusión: Es importante un alto índice de sospecha. El tratamiento se debe individualizar en cada paciente.
\end{abstract}

Palabras Clave: Bilioma. Perforación espontánea de la vía biliar. Peritonitis biliar.

\begin{abstract}
Introduction: Spontaneous perforation of the common bile duct is rare. The cause is usually unknown, although it is sometimes related to the malformation of the bile duct. Clinical case: Female of 1 year, with abdominal distention, hyporexia and fever, tomography with ascites, surgical findings included retroperitoneal bilioma, peritonitis and perforation of common bile duct. Block resection and primary hepato-duodenal anastomosis were performed. Discussion: Spontaneous perforation of the common bile duct tend to evolve insidiously and delay in diagnosis is frequent. Surgical management is decisive, and there are different techniques described. Conclusion: A high index of suspicion is important, treatment must be individualized according to each patient.
\end{abstract}

Key Words: Bilioma. Spontaneous perforation of the biliary tract. Biliary peritonitis.

\footnotetext{
Correspondencia:

*Cristhian Godínez-Borrego

Eurípides, 1635-8

Col. Residencial El Refugio

Fecha de recepción: 11-12-2018

Cir Cir. 2020;88(2):211-214

C.P.76146, Santiago de Querétaro, Qro., México

Fecha de aceptación: 14-06-2018

E-mail: drcggodinez@gmail.com

DOI: $10.24875 / C I R U .19000957$

Contents available at PubMed

www.cirugiaycirujanos.com

0009-7411/@ 2019 Academia Mexicana de Cirugía. Publicado por Permanyer. Este es un artículo open access bajo la licencia CC BY-NC-ND (http://creativecommons.org/licenses/by-nc-nd/4.0/).
} 


\section{Introducción}

La perforación espontánea de la vía biliar es un evento raro en pediatría, con pocos casos reportados en la literatura internacional. La causa no es del todo conocida y la teoría más aceptada involucra a la malformación de la unión pancreaticobiliar y lo que esta ocasiona'. Usualmente se presenta como distención abdominal asociada a ictericia e hiporexia, y en pocas ocasiones se puede presentar como un cuadro de abdomen agudo. Los estudios diagnósticos son poco sensibles o específicos, y el tratamiento en todos los casos es quirúrgico. El procedimiento dependerá de la condición del paciente al ingresar a quirófano y del grado de inflamación de las estructuras circundantes ${ }^{2}$.

\section{Presentación del caso}

Niña de 1 año, sin antecedentes, que presenta cuadro de distención abdominal acompañado de náuseas con vómito y evacuaciones disminuidas en consistencia, manejado como cuadro infeccioso gastrointestinal. De evolución insidiosa, al séptimo día se agregan fiebre, deshidratación e irritación peritoneal. Se protocoliza estudio con laboratorios, que muestran leucocitosis leve sin neutrofilia. La tomografía computarizada evidencia líquido libre en la cavidad y colección subdiafragmática derecha con septos finos (Fig. 1). Se realiza laparotomía en la que se reportan como hallazgos $250 \mathrm{ml}$ de líquido ictérico libre en la cavidad, adherencias laxas subhepáticas, peritonitis intensa e impresión biliar en el retroperitoneo cerca del colédoco (Fig. 2). Se realiza colangiografía transoperatoria en la que se identifica fuga biliar en la porción retroduodenal del colédoco (Fig. 3), por lo que se diseca e identifica una perforación puntiforme de $0.5 \mathrm{~cm}$ en la cara posterior, a $1 \mathrm{~cm}$ de su inserción con el páncreas. Se decide resección en bloque del colédoco y de la vesícula biliar, y se realiza una anastomosis hepatoduodenal. La evolución clínica posoperatoria fue satisfactoria, logrando su egreso a los 7 días del procedimiento. Con seguimiento posoperatorio de 6 meses, al momento actual está asintomática.

\section{Discusión}

La perforación espontánea de la vía biliar es un evento patológico raro en pediatría. Se observa con mayor frecuencia en pacientes menores de 20 semanas de vida, con una razón de presentación por sexo

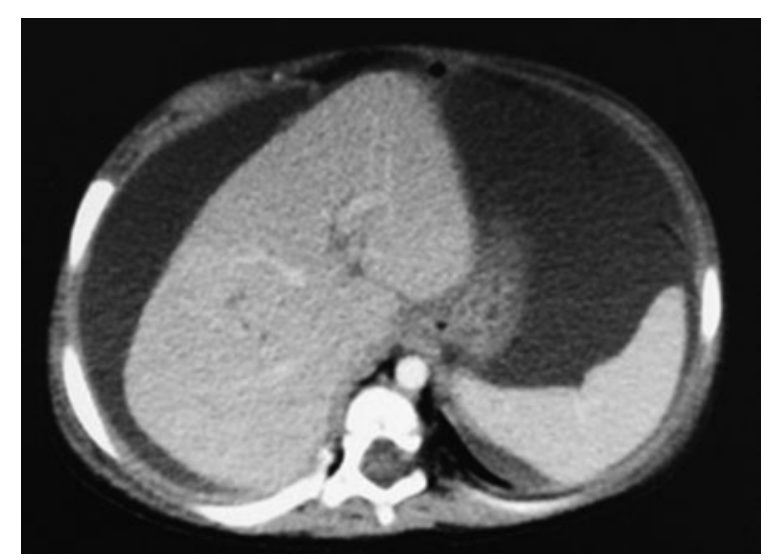

Figura 1. Tomografía computarizada de abdomen en la que se aprecia el líquido libre en la cavidad abdominal.

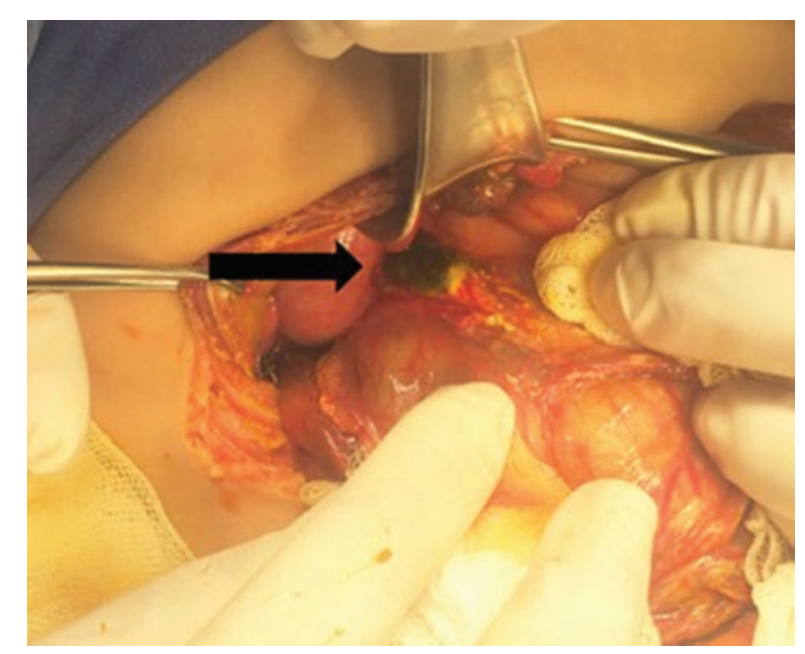

Figura 2. Imagen del bilioma retroperitoneal.

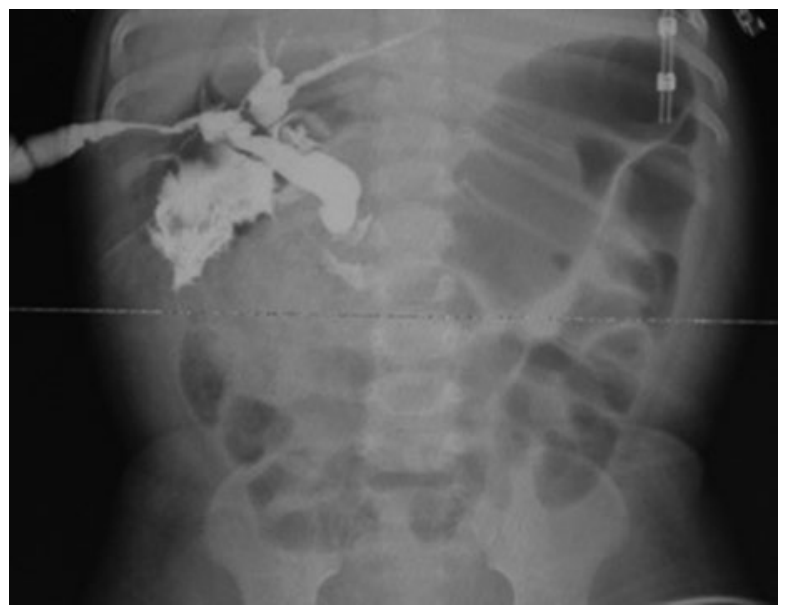

Figura 3. Colangiografía transoperatoria en la que se observa fuga del material de contraste.

de $1: 1^{3}$ y una incidencia aproximada de 1.5 en $1,000,000$ de recién nacidos vivos. Durante los últimos 15 años solo se han reportado 90 casos $^{4}$. La mayoría de los pacientes afectados suelen ser previamente 
sanos y por lo general no cuentan con antecedentes prenatales o posnatales de importancia ${ }^{5}$.

Desde el punto de vista etiopatológico, la causa más frecuente es la idiopática ${ }^{6}$, que se cree asociada a una malformación del colédoco en su desembocadura a nivel duodenal, que condiciona reflujo de enzimas pancreáticas, debilidad de la pared y predisposición a la perforación? ${ }^{7}$ Los cálculos biliares, el traumatismo, la intervención quirúrgica previa y los trastornos congénitos, como el quiste de colédoco, se presentan en menor medida ${ }^{8}$.

El sitio usual de perforación es la cara anterior del colédoco, cerca de la unión con el conducto cístico, lo que tiende a generar colección intraabdominal y peritonitis biliar ${ }^{9}$. Aquellas que se producen en el aspecto posterior del conducto se consideran raras, condicionan colección retroperitoneal y a su vez retraso en el diagnóstico, que en la mayoría de los casos se realiza al momento de la exploración quirúrgica ${ }^{10}$.

El cuadro clínico se divide usualmente en dos: una forma insidiosa, que representa el $80 \%$ de los casos y se caracteriza por distensión abdominal progresiva, hiporexia, ictericia, acolia y coluria; o una forma con síntomas agudos, que representa el $20 \%$ restante y se caracteriza por irritabilidad marcada, distensión abdominal, vómitos, fiebre e irritación peritoneal.

La biometría hemática suele ser inespecífica, aunque en estadios avanzados de la enfermedad tiende a haber leucocitosis y neutrofilia" ${ }^{11}$. Se ha documentado que en estos casos el ultrasonido abdominal muestra unos conductos biliares intrahepáticos y extrahepáticos normales, con presencia de líquido libre en la cavidad abdominal que en ocasiones se asocia a lóculos. En cambio, la tomografía sí logra evidenciar defectos en la pared, aunque se encuentra limitada por la necesidad de administrar contraste intraveno$\mathrm{so}^{12}$. El estudio más sensible y específico es el gamma grama hepático y de vía biliar. Otra modalidad es la paracentesis diagnóstica, que revela la presencia de bilis libre en la cavidad abdominal ${ }^{13}$.

Se han propuesto varias modalidades terapéuticas, que van desde la observación hasta la derivación biliodigestiva. El drenaje peritoneal simple o de la vía biliar con colocación de sonda en T se reporta como tratamiento de elección en casos no complicados, con buenos resultados según lo descrito en la literatura actual ${ }^{14}$, y la causa más común de falla en esta modalidad es la obstrucción distal del colédoco por lodo biliar e inflamación, que condiciona un aumento de la fuga biliar y la dificultad para el cierre. Otra técnica más compleja es la resección de los tejidos inflamados y la derivación biliodigestiva al duodeno o al yeyuno ${ }^{15}$. La decisión va a depender de las características clínicas del paciente en el momento del diagnóstico y del grado de inflamación de la región periportal ${ }^{16}$. Una de las complicaciones del drenaje como tratamiento único son las atresias adquiridas o la estenosis de la vía biliar ${ }^{17}$. Otras complicaciones asociadas no tan frecuentes son la colangitis, la trombosis de la vena porta y el bilioma ${ }^{18}$. Es importante que el diagnóstico y la resolución quirúrgica se hagan de forma temprana para disminuir el riesgo de complicaciones en el paciente ${ }^{19}$. El pronóstico depende de la gravedad del cuadro clínico, de la inflamación que condiciona y del tiempo transcurrido hasta la resolución quirúrgica definitiva, con grados variables de morbilidad y mortalidad que se asocian directamente al manejo quirúrgico diferido ${ }^{20}$.

\section{Conclusión}

La perforación espontánea del colédoco es rara en pediatría. Es importante tener un alto índice de sospecha en pacientes que presentan peritonitis biliar. El estudio tomográfico ayuda a establecer el diagnóstico. Se han descrito varias técnicas quirúrgicas, que van desde el drenaje externo simple hasta la resección y la derivación biliodigestiva; la decisión se tomará teniendo en cuenta las condiciones generales del paciente y el grado de inflamación local en la región periportal. En nuestra revisión de casos encontramos de buenos a excelentes resultados para la vida y la función de estos pacientes independientemente de la opción quirúrgica optada y de las características del paciente.

\section{Conflicto de intereses}

Los autores declaran que no existen conflictos de intereses.

\section{Responsabilidades éticas}

Protección de personas y animales. Los autores declaran que para esta investigación no se han realizado experimentos en seres humanos ni en animales.

Confidencialidad de los datos. Los autores declaran que han seguido los protocolos de su centro de trabajo sobre la publicación de datos de pacientes.

Derecho a la privacidad y consentimiento informado. Los autores han obtenido el consentimiento informado de los pacientes y/o sujetos referidos en el 


\section{artículo. Este documento obra en poder del autor de correspondencia.}

\section{Bibliografía}

1. Prada NE, Zarate LA. Perforación espontánea de la vía biliar. Reporte de un caso y revisión de la literatura. Salud UIS. 2008;40:197-201.

2. Paramhans D, Shukla S, Grover J. Spontaneus perforation of the common bile duct in an adult. Indian J Surg. 2003;75(Suppl 1):S376-8.

3. Alonso-Hernández MA, Ávila-Zaragoza LM, Jiménez-Urueta PS, Castañeda-Ortiz RA, Gutiérrez-Escobedo J. Perforación espontánea de la vía biliar en una preescolar. Acta Pediatr Mex. 2011;32:136-9.

4. Amberger M, Burton N, Tissera G, Baltazar G, Palmer S. Spontaneous common bile duct perforation - a rare clinical entity. Int J Surg Case Rep. 2018;46:34-7.

5. Hasegawa T, Udatsu Y, Kamiyama M, Kimura T, Sasaki T, Okada A et al. Does pancreatico-biliary maljunction play a role in spontaneous perforation of the bile duct in children? Pediatr Sug Int. 2000;16:550-3.

6. Jain S, Jain M, Kaur D, Shukla L. Management of spontaneous perforation of the bile duct in an infant in a semi-urban setup: a case report. Malays J Med Sci. 2012;19:73-5.

7. Baeza-Herrera C, Medellín-Sierra UD, Sanjuán-Fabián H, Jiménez-González N, García-Cabello LM. Malformación del sistema pancreático-biliar como causa de perforación espontánea de la vía biliar extrahepática. Gac Med Mex. 2006;142:79-80.

8. Sai Prasad TR, Hon Chui C, Low Y, Li Chong C, Sundfor Jacobson A Bile duct perforation in children: is it truly spontaneus? Ann Acad Med Singapore. 2006;35:905-8.

9. Ishii K, Matsuo K, Seki H, Yasaui N, Sakata M, Shimido A, et al. Retroperitoneal biloma due to spontaneous perforation of the left hepatic duct. Am J Case Rep. 2016;17:264-7.
10. Sahnoun L, Belghith M, Jallouli M, Maazoun K, Mekki M, Ben Brahim M, et al. Spontaneous perforation of the extrahepatic bile duct in infancy: report of two cases and literature review. Eur J Pediatr. 2007;166:173-5.

11. Suleman-Malik H, Arshad-Cheema H, Fayyaz Z, Almas-Hashmi M, Fayyaz Z, Almas Hasmi M, et al. Spontaneous perforation of bile duct, clinical presentation, laboratory work up, treatment and outcome. J Ayub Med Coll Abbottabad. 2016;28:518-22.

12. Kumar-Mohanty S, Mahapatra T, Kumar Behera B, Acharya B, Kumar S, Ranjan-Dash R, et al. Spontaneous perforation of common bile duct in a young female: an intra-operative surprise. Int J Surg Case Rep. 2017;35:17-20.

13. Bjorn N, Bjorn M, Qvist N. Spontaneous bile duct perforation in a newborn. J Pediatr Surg Case Rep. 2017;26:46-7.

14. Jeanty C, Derderian SC, Hirose S, Lee H, Padilla BE. Spontaneous biliary perforation in infancy: management strategies and outcomes. J Pediatr Surg. 2015;50:1137-41.

15. Evans K, Marsden N, Desai A. Spontaneous perforation of the bile duct in infancy and childhood: a systematic review. J Pediatr Gastroenterol Nutr. 2010;50:677-81.

16. Goel P, Jain V, Manchanda V, Sengar M, Ranu Gupta C, Mohta A. Spontaneous biliary perforations: an uncommon yet important entity in children. J Clin Diagn Res. 2013;7:1201-6.

17. Pulat $\mathrm{H}$, Karakose $\mathrm{O}$, Fatih Benzin $M$, Zafer Sabuncuoglu $M$, Cetin $\mathrm{R}$ A rare cause of acute abdomen: spontaneous common hepatic duct perforation. Ulus Trauma Acil Cerrahi Derg. 2016;22:103-5.

18. Laway M, Bakshi I, Shah M, Paray S, Sadiq Malla M. Biliary peritonitis due to spontaneous perforation of choledochus: a case report. Indian J Surg. 2013;75(Suppl 1):S96-8.

19. Hadi Imanieh M, Mowla A, Zohouri D, Reza Forootan H, Karimi M. Spontaneous perforation of the common bile duct with eosinophilia in an 18-month-old girl: a case report and review of literature. Med Sci Monit. 2006;12:CS31-3.

20. Sharma $\mathrm{C}$, Desale J, Waghmare $\mathrm{M}$, Shah $\mathrm{H}$. A case of biliary peritonitis following spontaneous common bile duct perforation in a child. Euroasian J Hepatogastroenterol. 2016;6:167-9. 